\title{
Le corps à fleur de mots, sous la direction de Elisa Girardini et Genevieve Henrot
}

\section{Rampazzo}

\section{Q OpenEdition}

12 Journals

\section{Edizione digitale}

URL: http://journals.openedition.org/studifrancesi/36722

DOI: 10.4000/studifrancesi.36722

ISSN: 2421-5856

\section{Editore}

Rosenberg \& Sellier

\section{Edizione cartacea}

Data di pubblicazione: 1 juillet 2005

Paginazione: 201-202

ISSN: 0039-2944

\section{Notizia bibliografica digitale}

Rampazzo, «Le corps à fleur de mots, sous la direction de Elisa Girardini et Genevieve Henrot», Studi Francesi [Online], 145 (XLIX | I) | 2005, online dal 30 novembre 2015, consultato il 20 avril 2021. URL: http://journals.openedition.org/studifrancesi/36722 ; DOI: https://doi.org/10.4000/studifrancesi. 36722

Questo documento è stato generato automaticamente il 20 avril 2021.

\section{(c) (1)}

Studi Francesi è distribuita con Licenza Creative Commons Attribuzione - Non commerciale - Non opere derivate 4.0 Internazionale. 


\title{
Le corps à fleur de mots, sous la direction de Elisa Girardini et Genevieve Henrot
}

\author{
Rampazzo
}

\section{NOTIZIA}

AV. VV., Le corps à fleur de mots, sous la direction de ELISA GIRARDINI et GENEVIEVE HENROT, Padova, Unipress, 2004, pp. 181.

1 Il rapporto tra corpo e scrittura, filo conduttore che lega l'uno all'altro gli otto saggi di questo volume, è suggerito dall'originale calligramma in copertina. Il tema è attualmente di forte e vivissimo interesse, al punto tale da fecondare studi e ricerche nei campi più disparati, dalla storia alla sociologia, dalla psicoanalisi alla linguistica. Dopo l'introduzione generale delle due curatrici, le tre parti che costituiscono il volume analizzano secondo diverse prospettive la descrizione del corpo e il valore attribuitogli nella letteratura francese dal XVI secolo ad oggi. In questa sede, l'attenzione sarà focalizzata sugli ultimi cinque saggi, riguardanti la seconda metà del XX secolo.

2 La parte centrale della raccolta è dedicata a tre autori del XX secolo, e articolata in tre studi che partono da alcuni presupposti mutuati dalle più recenti teorie psicanalitiche: quelle post-freudiane per Elisa GIRARDINI (Caligula ou le corps (dé)figuré, pp. 71-88), e il concetto di "Moi-peau" formulato da Didier Anzieu per Geneviève HENROT (Pascal Quignard et la peau de Marsyas, pp. 89-111) e per Sara BASTESIN (Itinéraires génétiques du corps chez Henry Bauchau, pp. 113-133). Confrontando le due versioni maggiori del Caligula di Camus, quella del 1941 e quella del 1958, il primo di questi tre saggi vi rileva la demolizione della nozione di personaggio e il ruolo centrale, di vero e proprio cardine, assegnato al corpo. Quello dell'imperatore Caligola innanzitutto, un corpo in iperbole, enfatizzato e capace di rivestire molteplici ruoli, ma anche un corpo in litote, come rivela tutta una serie di procedimenti di svilimento, di passivazione sintattica, di 
animalizzazione e addirittura di reificazione. In secondo luogo, il corpo della sua giovane sorella Drusilla, la cui rilevanza viene messa nel giusto rilievo da questo studio. Anch'esso può essere considerato sia in litote sia in iperbole: da un lato infatti la presenza di Drusilla, che già nella prima versione era solo allusiva, risulta ulteriormente minimizzata nella seconda, attraverso l'eliminazione non solo della sua ombra, che viene introiettata e intellettualizzata, ma anche e persino del suo nome, che appare un'unica volta, nel finale, ma solo per essere negato, facendo così passare il personaggio da un "elle" a un "ce", quindi ad un "ça" reificato e annientato, e infine all'assoluta vacuità di un "rien"; dall'altro lato, si può constatare che questa presenza non venga completamente eliminata dal testo, ma vi occupi invece una posizione di prestigio.

3 Su un autore ancor più recente, Pascal Quignard, verte il secondo studio. L'analisi dei romanzi di questo scrittore, tra i quali Carus (1979), Le Salon du Wurtemberg (1986), La Haine de la musique (1996) e Vie secrète (1998), consente di tracciare l'itinerario, apparentemente paradossale, che dall'iniziale esaltazione entusiastica della musica, arte per eccellenza perché prima e prelinguistica, in quanto basata sui sensi più arcaici dell'uomo, ovvero le percezioni sonore del feto, porta lo scrittore ad un profondo e radicato odio nei suoi confronti, a favore della letteratura. In un primo momento, infatti, dopo la lacerazione traumatica del primo involucro psichico comune alla madre $\mathrm{e}$ al figlio, della nascita, la musica sembra capace di offrire una seconda pelle più dura $\mathrm{e}$ resistente, una metaforica corazza che rievoca quella reale, di una tartaruga, con la quale, secondo il mito greco, Ermes costruì il primo strumento musicale. In questa prima fase quest'arte, insegnata dal padre organista al piccolo Charles Chenogne, protagonista de Le Salon du Wurtemberg, rappresenta il dono paterno in grado di sostituire quello materno, la lingua francese. Nel romanzo, il carattere assente e silenzioso della madre, avara di parole, affetto e carezze, infligge al protagonista delle sofferenze insormontabili, scorticandolo vivo proprio come accade al leggendario Marsia, inventore della musica secondo il mito greco. Ma a poco a poco passione ed esaltazione si mutano in odio viscerale. Le ragioni di tale cambiamento radicale vanno individuate in tre fattori principali: l'imporsi della musica come la lingua paterna, dinastica, ancestrale, impregnata del super-Io paterno; il fatto che, avendo prestato la sua ipocrita collaborazione al genocidio perpetrato nei campi di concentramento nazisti, essa sia stata organo e strumento di un'autorità coercitiva; ed infine il profondo divario che separa l'alto valore della musica barocca dalla profonda decadenza di quella contemporanea, che con la sua ubiquità ossessiva, le sue melodie insistenti, il volume eccessivo e l'alta fedeltà fredda e maniacale assedia e invade l'ascoltatore. Partendo da questi presupposti, non è poi così paradossale per il protagonista, divenuto ormai adulto, abbandonare la musica e trovare un altro involucro, costituito di parole, e rappresentato dalla lingua materna, il francese, addomesticata a furia di esercizio, tenacia e volontà. La parabola della maturazione del protagonista si conclude con la conquista di un Io sano e autonomo e col rammendo della pelle originaria, quella della lingua materna, ricucitura resa possibile dall'effetto cicatrizzante della letteratura.

4 Lo studio conclusivo della seconda parte della raccolta analizza il legame tra corpo e parola scritta nell'opera di Henry Bauchau e assegna un ruolo decisivo e fondamentale alla psicoanalisi e alla scrittura, evidenziando l'intimo legame che le unisce. In Bauchau bambino, le sofferenze causate dalla lacerazione del primo involucro psichico e dall'assenza del calore materno impediscono di comprendere e di esprimere la parte più profonda dell'Io. Nell'età adulta, la scoperta della psicoanalisi consente la 
liberazione della scrittura, la quale, dopo aver superato un'iniziale resistenza fisica dell'autore causata dalla permanenza dei fantasmi e dei divieti del passato, ristabilisce il contatto tra i sensi e lo spirito, permettendo finalmente l'espressione dei labirinti interni dell'Io, e sostituendo la prima pelle lacerata con una seconda più resistente. Nella parte finale del saggio, la focalizzazione degli elementi metaforici, allegorici e onirici e la messa in evidenza dei più frequenti topoi dei sogni dell'autore assegnano al rêve il ruolo di crogiolo in cui si condensa l'itinerario genetico della scrittura.

5 I due contributi che compongono la terza parte vertono anch'essi su autori recentissimi, appartenenti all'ultimo decennio del XX secolo e addirittura agli inizi del XXI, ma optano per una prospettiva di tipo sociologico. In Petites filles ratées et garçons manqués dans le roman féminin contemporain (pp. 137-151), Anne SIMON e Christine DÉTREZ analizzano la figura della bambina "mal riuscita" che compare nei romanzi femminili a partire dagli anni ' 90 , e ne mettono in evidenza l'azione di rottura rispetto al modello positivo, virtuoso e perbene della tradizione letteraria, nonché quella di sovversione dell'ordine sociale. Rifiutando il modello materno, queste bambine mal riuscite cercano di attirare l'attenzione del padre tentando di diventare come lui, ed uniformandosi così ad archetipi prettamente maschili. Ciò giustifica il carattere androgino che esse rivelano nell'aspetto fisico, negli atteggiamenti, nella scelta dei giochi e dei vestiti e nel linguaggio. Diretta conseguenza dei loro insuccessi, errori e rivolte, e indice di uno scarto rispetto alla norma, è lo sconvolgimento dell'ordine sociale, sovvertimento che i loro genitori cercano di contrastare ricorrendo alla medicina, somministrando ad esempio alle piccole ribelli delle iniezioni di ormoni femminili. Solo l'azione salvifica dell'arte permetterà loro, divenute ormai adulte, di creare se stesse, trovare il loro posto e ricomporre la loro identità, risolvendo le inettitudini e le deficienze del loro corpo in una sorta di metamorfosi finale di quest'ultimo in libro.

6 Se l'argomento della sessualità non è che una delle tante tematiche affrontate da Anne SIMON e Christine DÉTREZ, nell'intervento di Jean-Michel DEVÉSA (Le corps glorieux de Catherine M., pp. 153-178) esso diventa invece l'elemento principale e dominante. DEVÉSA si propone di riabilitare il romanzo autobiografico La vie sexuelle de Catherine M. (2002) di Catherine Millet, a suo avviso ingiustamente bollato come pornografico, attribuendone l'eclatante successo non tanto al fortunato connubio tra una riuscita operazione editoriale e la decadenza dei costumi e dei valori, quanto piuttosto all'efficacia dello stile che, conquistato con ostinato accanimento, riesce senza affettazione né tabù a esprimere mirabilmente la sessualità. L'opera, testimonianza quasi clinica delle esperienze erotiche della protagonista, traccia l'itinerario che quest'ultima compie per arrivare a una migliore accettazione di sé e alla conquista di un corpo capace sia di dare sia di ricevere piacere. Dallo status iniziale di bambola meccanica, di corpo-macchina annientato e reificato dalla pratica ossessiva di un eros di gruppo e puramente fisico, essa giunge infine a quello che l'autore ritiene un surrogato del "corpo glorioso", vale a dire un corpo carnale che riunisce in sé tutte le facoltà e le debolezze umane da un lato, e le potenzialità in grado di conferirgli un carattere spirituale dall'altro. Il saggio esalta infine la virtù catartica dell'atto dello scrivere, strumento di liberazione dai fantasmi e dal terrore relativo alla precarietà dell'esistenza e all'azione corrosiva del tempo, e attribuisce alla scrittura il potere di trasmutare e sublimare il fango umano in oro letterario.

7 Il volume si conclude con una breve presentazione di ciascuno dei nove autori. 